\title{
Trypanosoma cruzi: Maintenance in Culture Modify Gene and Antigenic Expression of Metacyclic Trypomastigotes
}

\author{
Víctor T Contreras ${ }^{+}$, Ana Rita De Lima, Gelu Zorrilla
}

\author{
Centro de Biología Molecular de Parásitos, Facultad de Ciencias de la Salud, Universidad de Carabobo, \\ Valencia, Venezuela
}

In this study we examined whether the maintenance of Trypanosoma cruzi by long-time in axenic culture produces changes in gene expression and antigenic profiles. The studies were made with a Dm30L-clone from a low-virulent strain and a non-cloned virulent EP-strain of T. cruzi. Both parasites were maintained, for at least seven years, by successive alternate passage triatomine/mouse (triatomine condition), or by serial passage in axenic medium (culture condition). The comparison of the $\left[{ }^{35}\right.$ S]methionine metabolic labeling products of virulent and non-virulent parasites by $2 D-S D S-P A G E$, clearly indicates that the expression of metacyclic trypomastigotes (but not of epimastigotes) proteins have been altered by laboratory maintenance conditions. Western blot analysis of EP and Dm3OLepimastigotes using a serum anti-epimastigotes revealed that although most of antigens are conserved, four antigens are characteristics of triatomine condition parasites and three other are characteristics of culture condition parasites. Anti-metacyclics serum revealed significative differences in EP-and Dm30L-metacyclic trypomastigotes from triatomine condition. However, avirulent metacyclic forms were antigenically very similar. These results suggest that besides a possible selection of avirulent subpopulation from T. cruzi strains genetically heterogeneous when maintained by long time in axenic culture, changes in virulence might be due to post-translational modifications of the antigens induced by the absence of the natural alternability (vertebrate-invertebrate) in the life-cycle of $\mathrm{T}$. cruzi

Key words: gene expression - metabolic labeling - metacyclic - Trypanosoma cruzi

Trypanosoma cruzi, the ethiological agent of Chagas'disease, is a protozoan parasite whose genetic heterogeneity might result in different patterns of virulence, pathogenicity and infectivity to animals (Carneiro et al. 1991). In nature, the infection by this parasite is initiated when metacyclic trypomastigotes eliminated in the excreta of triatomine vectors enter the mammalian host and invade a variety of cell types. Despite the obvious importance of understanding at the molecular level the reasons underlying the virulence of metacyclic forms, very few studies have been reported, mainly because this developmental form is eliminated in faeces of insect vector in tiny amounts.

Due to the difficulties in producing an appreciable number of metacyclic trypomastigotes from

This work was supported by the Venezuelan agencies: CONICIT Projects S1-97000664; RP-IV-097; CODECIHT-UC FCS-95018, FCS-97018. Aga Venezolana and CORPOVEN.

${ }^{+}$Corresponding author. Fax: +58-41-673342. E-mail: convictu@cantu.net

Received 12 February 1998

Accepted 13 July 1998 triatomine urine (Garcia et al. 1984) culture-derived metacyclic $T$. cruzi have been used. However, it has been reported that the maintenance by long time in culture modifies biological properties of this parasite (Chiari 1974). Therefore, the extrapolation of results obtained using culture-derived metacyclic forms to insect-born metacyclic trypomastigotes have been questioned (Villalta \& Kierszenbaum 1987). This problem can be avoided comparing in vitro induced metacyclic trypomastigotes whose biological properties are equivalents.

In this study, we took advantage of the fact that the metacyclogenesis process of $T$. cruzi can be mimicked in vitro using a chemically defined differentiation medium (Contreras et al. 1985b). With this medium, biochemical analysis of metacyclic forms is feasible and indeed has allowed the identification of stage-specific gene expression products, as well as the association between gene products and acquisition of metacyclic-specific trypomastigote biological properties (Contreras et al. 1985a,c, Goldenberg et al. 1987, Bonaldo et al. 1988).

Previous results from our laboratory with the virulent-EPstrain and the low-virulent Dm30Lclone of $T$. cruzi have shown that their long time maintenance in axenic culture might lead to loss 
of synchronization of morphogenetic events that would occur in the vertebrate host. Culture-derived metacyclic forms had decreased their capacity to invade cells and whenever becoming intracellular were destroyed inside phagolysosomes (Contreras et al. 1994). There is increasing evidence that the entry of T. cruzi invasive forms into mammalian cells involves glycoconjugates-dependent attachment followed by parasite internalization (Andrews et al. 1984, Yoshida et al. 1990, 1997, Schenkman et al. 1993, Ruiz et al. 1993, Ramirez et al. 1993, Vermelho \& Meirelles 1994, Burleigh \& Andrews 1995). The glycoconjugates, including glycolips and glycoproteins may therefore play a role important in the virulence of the parasites. Recently, Chuenkova and Pereira (1995) demonstrated that an enzyme expressed by trypomastigotes might be implicated in the increase of the parasite virulence. Hence, the molecular basis of the virulence changes described above are not yet clear. Our working hypothesis is that the loss of virulence of $T$. cruzi can be associated to molecular changes in the metacyclic forms.

In this study, we provide evidence suggesting that the loss of virulence, by maintenance of $T$. cruzi in culture might associated to changes in parasite gene expressions.

\section{MATERIALS AND METHODS}

Parasites and maintenance conditions - Two isolates of T. cruzi were used: a clone from lowvirulent strain (Dm30L) isolated from opossum and a non-cloned virulent strain (EP) isolated from a fatal human case. Both parasites were maintained, for at least seven years, by successive triatomine/ mouse passages (triatomine condition) or by serial passages in axenic medium (culture condition) as previously described (Contreras et al. 1994).

Preparation of epimastigotes - To obtain epimastigotes, parasites in late exponentially growing phase in LIT medium (Castellani et al. 1967) were harvested by centrifugation at $8,500 \mathrm{xg}$, for $20 \mathrm{~min}$ and then washed twice with PBS 0.15 M $\mathrm{pH}$ 7.2. Epimastigotes of triatomine condition were obtained from parasites kept for no more than two months in culture.

Harvesting of metacyclic forms - Metacyclic trypomastigotes were induced by the technique of Contreras et al. (1985b). Briefly, epimastigotes from the exponentially growing phase in LIT medium were harvested by centrifugation at $8.500 \mathrm{xg}$ and incubated for $2 \mathrm{hr}$ in artificial triatomine urine (TAU, $190 \mathrm{mM} \mathrm{NaCl}, 17 \mathrm{mM} \mathrm{KCl}, 2 \mathrm{mM} \mathrm{CaCl}_{2}$, $2 \mathrm{mM} \mathrm{MgCl}_{2}, 8 \mathrm{mM}$ phosphate buffer $\mathrm{pH}$ 6.0) in a density of $5 \times 10^{8} \mathrm{cell} / \mathrm{ml}$. Thereafter, the parasites were incubated in TAU3AAG medium (TAU supplemented with $10 \mathrm{mM}$ L-proline, $50 \mathrm{mM} \mathrm{L-}$ glutamate, $2 \mathrm{mM}$ L-aspartate, $10 \mathrm{mM}$ glucose) to a final concentration $3 \times 10^{6} \mathrm{cell} / \mathrm{ml}$ in a final volume of $70 \mathrm{ml}$ in Roux flask. They were incubated at $27^{\circ} \mathrm{C}$ for $72 \mathrm{hr}$, then centrifuged at $10,000 \mathrm{xg}$, resuspended in TAU medium, treated for $30 \mathrm{~min}$ at $37^{\circ} \mathrm{C}$ with fresh guinea pig serum, and separated on DEAE cellulose (Sousa 1983) as previously described (Contreras et al. 1994). Metacyclic trypomastigotes from triatomine condition were obtained from epimastigote forms with no more than two months in culture. Alternatively, insectborn metacyclic forms were obtained from the urine of Rhodnius prolixus infected with T. cruzi (Garcia et al. 1984).

Infection of mice - Groups of 9-18 female NMRI mice were inoculated with insect or TAU3AAG-derived metacyclic forms. Three days post-inoculation, blood was examined to determine parasitaemia (Brener 1962). Sub-patent infections (30 days post-inoculation) were established by three separate xenodiagnosis with ten 4th-instar nymphs of $R$. prolixus at two days intervals, and the insects were examined 30,45 , and 60 days after infection. Hemoculture was made to complement the xenodiagnosis.

In vivo labeling of parasites with $\left[{ }^{35} \mathrm{~S}\right] \mathrm{me}$ thionine - Metabolic labeling of epimastigotes or metacyclic trypomastigotes was carried out with $\left[{ }^{35}\right.$ S]methionine (1.-100 Ci/mMol, New England Nuclear) as previously described (Contreras et al. 1985a). Metabolically labeled parasites were resuspended in $0.5 \mathrm{ml}$ of lysis solution $(150 \mathrm{mM}$ $\mathrm{NaCl}, 10 \mathrm{mM}$ Tris/HCl pH 7.4, 1 mM EDTA, 1 $\mathrm{mM}$ Iodoacetamide, $1 \mathrm{mM}$ 1.10-phenanthroline, $0.1 \mathrm{mM}$ PMSF, $0.5 \mathrm{mM} \mathrm{Na-p-tosil-L-Lysine}$ chloro-methyl ketone (TLCK), $0.5 \mathrm{mM} \mathrm{N}$-TosilL-phenylalanine chloromethyl ketone (TPCK), 0.1 $\mathrm{mM}$ trans-epoxysuccinyl-L-leucylamido (4guanidino)-butane (E-64), $25 \mu \mathrm{M}$ Leupeptin, Antipain $10 \mu \mathrm{g} / \mathrm{ml}, 1 \% \mathrm{NP}-40$ ) homogenized for $3 \mathrm{~min}$ at room temperature and centrifuged $10 \mathrm{~min}$ at $12,000 \mathrm{xg}$. The supernatant was immediately processed for electrophoresis, as previously described (Contreras et al. 1985a).

Protein analysis - The proteins were analyzed by two-dimensional SDS-PAGE (2D-SDS-PAGE) essentially as described by O'Farrell (1975). Aliquots containing 4 or $6 \times 10^{5} \mathrm{cpm}$ of metabolically labeled proteins were resuspended in 30 $45 \mu \mathrm{l}$ of $9.5 \mathrm{M}$ urea, $2 \%$ ampholines $(1.6 \%$ ampholines $\mathrm{pH}$ 5-7, 0.4\% ampholines $\mathrm{pH} 3-10$, Sigma), 5\% 2-mercaptoetanol and 2\% NP-40. The first dimension was an isoelectric focusing $(4,800$ $\mathrm{V} / \mathrm{hr}$ ) and the second-dimension was performed by SDS-PAGE on 7-15\% linear gradient gels (Laemmli 1970). After electrophoresis the gels 
were processed for fluorography (Laskey \& Mills 1977) and autoradiographed at $-70^{\circ} \mathrm{C}$ using Agfa films and Dupont Cronex-Plus intensifying screen.

Western blot analysis - Whole cell lysates of T. cruzi (EP, Dm30L) epimastigotes and metacyclic forms from triatomine and culture conditions, containing the same protease inhibitor cocktail described above, were electrophoretically separated by SDS-PAGE according to Laemmli (1970) on $7-15 \%$ linear gradient gels. The concentration of proteins was determined according to Spector (1981). Aliquots containing $4 \mu \mathrm{g}$ of proteins per lane were electrophoresed at $10 \mathrm{mAmp}$ during 4 $\mathrm{hr}$ and blotted onto nitro-cellulose membrane at 0.65 volts $/ \mathrm{cm}^{2}$ for $1.5 \mathrm{hr}$ in a Trans Blot apparatus (LKB) according to Towbin et al. (1979).

After blocking in Tris Buffer Solution $(10 \mathrm{mM}$ Tris- $\mathrm{HCl} \mathrm{pH} 7.4,150 \mathrm{mM} \mathrm{NaCl}$ ) containing nonfat dried milk, the nitrocellulose membrane was incubated overnight at $4^{\circ} \mathrm{C}$ with 1:500 dilution of rabbit sera. After six washes with TBS, membranes were incubated ( $2 \mathrm{hr}$ at room temperature) with 1:3000 dilution of peroxidase-conjugate goat antirabbit total immunoglobulins and developed by LumiGLO (Kirkegaard \& Perry Laboratories) chemiluminescense system according to the protocol supplied by the manufacturer (Leskawa et al. 1993). Finally, the blots were wrapped in plastic wrap and luminographed using Agfa films and DuPont Cronex-Plus intensifying screen. The molecular weight was calculated by a calibration curve obtained with rainbow molecular weight markers (SIGMA).

Antisera production - Epimastigotes or in vitro induced metacyclic trypomastigotes of T. cruzi EPtriatomine condition were used to prepare antiepimastigote serum (anti-Epi) and anti-metacyclic serum (anti-Meta), respectively. Twenty mg wet weight of parasites were resuspended in $2 \mathrm{ml}$ of Freund's complete adjuvant and injected subcutaneously in the scruff of the neck of New Zealand rabbits weighing $3 \mathrm{~kg}$. Fifthteen days later, the rabbits were inoculated two times intramuscularly at eight day intervals with $20 \mathrm{mg}$ of wet weight of parasites resuspended in $2 \mathrm{ml}$ of Freund's incomplete adjuvant. Following this, they were inoculated intravenously three times at $48 \mathrm{hr}$ intervals with $2 \mathrm{mg}$ of freeze-thawed parasites in Hanks'solution. Two weeks after the last inoculation the immune response was assayed by indirect immunofluorescense (Camargo 1966) with the homologous antigen. This procedure was repeated until a titer greater than 1:640 was obtained against the homologous antigen. The rabbits were bled and the antiserum obtained was kept in aliquots at $-70^{\circ} \mathrm{C}$.

Densitometric analysis - Protein profiles comparisons and molecular weight determinations corresponding to the bands detected after SDS-PAGE and immunoblots, were quantitated by densitometry using a Scanner BioRAD Mod. GPS- 670 and the data calculated with a Molecular Analyzer Software Ver. 1.2 The following radioactive (14C) molecular mass markers (Amershan) were used: myosin (200000), a-globulin (95000), bovine serum albumin (69000), ovoalbumin (46000), carbonic anhidrase (30000), protein A (21500) Citocrome B (14300). The rainbow markers (Sigma) were myosine (205000), b-galactosidase (116000), bovine serum albumin (66000), ovoalbumin (45000), carbonic anhidrase (29000) and aprotinin (6500).

\section{RESULTS}

Decrease in the virulence of metacyclic trypomastigotes of $\mathrm{T}$. cruzi by maintenance in culture - Metacyclic trypomastigotes derived from parasites maintained by different times in culture were inoculated in mice and the classical parameters of virulence were compared. Table shows the virulence of metacyclic trypomastigotes of EPstrain of T. cruzi from urine of triatomine or induced in TAU3AAG medium. Metacyclic forms from urine (0 days in culture) infected $100 \%$ of

TABLE

Virulence of Trypanosoma cruzi (EP) from TAU3AAG medium. Effect of time in culture

\begin{tabular}{|c|c|c|c|c|}
\hline $\begin{array}{l}\text { Time in } \\
\text { culture } \\
\text { (days) }\end{array}$ & $\begin{array}{c}\text { Infectivity } \\
(\%)\end{array}$ & $\begin{array}{c}\text { Prepatency } \\
\text { (means } \pm \text { s.d.) } \\
\quad \text { (days) }\end{array}$ & $\begin{array}{c}\text { Mortality } \\
(\%)\end{array}$ & $\begin{array}{c}\text { Survival } \\
\text { (means } \pm \text { s.d.) } \\
\text { (days) }\end{array}$ \\
\hline $0^{a}$ & 100 & $7 \pm 1$ & 100 & $26 \pm 1$ \\
\hline 20 & 100 & $9 \pm 1$ & 100 & $26 \pm 3$ \\
\hline 78 & 100 & $15 \pm 3$ & 44 & $33 \pm 2$ \\
\hline 94 & 100 & $14 \pm 5$ & 27 & $33 \pm 3$ \\
\hline 256 & 28 & ${ }^{3} 30^{b}$ & 0 & $>260$ \\
\hline
\end{tabular}

Inoculation ip of NMRI mice of 20 day of age (10,000 metacyclics/mouse). a: metacyclics from urine of Rhodnius prolixus; $b$ : parasitaemia subpatent determined by xenodiagnosis. 
the mice with a prepatent period of $7 \pm 1$ days, $100 \%$ mortality and survival of $26 \pm 1$ days. Mice infected with in vitro differentiated metacyclic trypomastigotes derived from parasites kept for 20 days in culture presented an increased prepatence period ( $9 \pm 1$ days). Metacyclic trypomastigotes derived from parasites maintained in culture for 78 and 94 days also infected $100 \%$ of mice, but with a significative increase in their prepatent periods (15 and 14 days) and the mortality of mice was drastically reduced $(44 \%$ and $27 \%$ ). Finally, the metacyclic trypomastigotes derived from parasites kept for 256 days in culture infected $28 \%$ of the mice without patent parasitaemia and no mortality.

Analysis of in vivo synthesized proteins by twodimensional SDS-PAGE - In our experimental conditions, metacyclic trypomastigotes generally incorporate more radioactivity than epimastigotes. In order to examine whether the specific activities showed by these parasites were associated with differences in the proteins synthesized by epimastigotes and metacyclic trypomastigotes, the metabolic labeling products were analyzed by two-dimensional gel electrophoresis. Analysis of proteins synthesized by epimastigotes is shown in Fig. 1. Fig. 1A shows the protein profile of the strain EP epimastigotes from triatomine condition. The comparison of this profile with the protein profile displayed by epimastigotes of the clone Dm30L from the same condition (Fig. 1C) shows very little changes, represented by the absence of some polypeptides (4, and group 10, Fig. 1A) and the lowering in intensity of other polypeptides (e.g. 5, groups 13, 14, 15 in Fig. 1C). When the profiles of parasites from the different strains maintained at culture condition were compared no differences could be seen (Figs 1B, 1D). Interestingly, the polypeptides more intensely labeled are in the range of 92.5 to $21.5 \mathrm{kDa}$ of $\mathrm{Mr}$ with $\mathrm{pI}$ values between 4.3 and 6.2 and belong to epimastigotes from culture condition. On the other hand, epimastigotes (Figs 1A , 1C) displayed a more complex protein profile than metacyclics (Figs 2A, 2C).

The comparison by 2D-PAGE of the protein synthesis patterns of metacyclic forms is shown in Fig. 2. Fig. 2A shows the protein profile of the virulent EP-strain from triatomine condition. This profile revealed several spots with a wide range of isoelectric points. A first acidic group, previously identified as being metacyclic-specific (Contreras et al. 1985a), is composed of at least four strongly expressed polypeptides. They were: polypeptide 1 (86/92 kDa, pI 4.3-4.6), polypeptide $2(82 / 85 \mathrm{kDa}$, pI $4.3-5.5)$, polypeptide 3 (72 $\mathrm{kDa}, \mathrm{pI} 5.6)$ and polypeptide $6(72 / 75 \mathrm{kDa}, \mathrm{pI} 4.8$
-5.3). A second group is composed of one polypeptide intensely expressed, polypeptide 4 (70/72 kDa, pI 5.3-5.7) and other with a lower intensity polypeptide $5(60 / 65 \mathrm{kDa}, \mathrm{pI} 4.3-4.8)$. A third group was formed by polypeptides barely detectable displaying an isoelectric point ranging from 4.6 -6.1 and with Mr between 46 and $65 \mathrm{kDa}$. A fourth group with lower intensity displaying an isoelectric point ranging from 4.2 -6.5 and with Mr between 30 and $46 \mathrm{kDa}$. In this last range of molecular weight other two polypeptides intensely expressed were detected; group 7 (45 kDa, and pIs values of $4.4,5.2,5.4)$ and polypeptide $8(40 \mathrm{kDa}$, pI 5.4). Finally a series of low-molecular mass polypeptides (polypeptide 12) barely detectable were identified in the region between $\mathrm{pI} 4.3$ and 5.6. The comparison of this profile with the protein profile displayed by the avirulent-EP metacyclic trypomastigotes (Fig. 2B) shows important changes, represented by a absence of several acidic polypeptides (1, 6, and 4, Fig. 2A), and lowering in intensity of other polypeptides $(2,3$, 5, 7, 8, Fig. 2B), and the appearance and increase in intensity of various another polypeptides belonging to fourth group described above $(9,10,11,12$ and 13, Fig. 2B). It is worth noting that the protein profiles displayed by non-infective metacyclic trypomastigotes (Fig. 2B) resembles those from epimastigotes (Fig. 1).

The comparison of metacyclic forms from triatomine conditions (Figs 2A, 2C) and their noninfective counterparts from culture conditions (Figs 2B, 2D) shows, for the latter, an increase in the complexity of the polypeptide patterns. Comparison of the profiles of metacyclic forms of the virulent EP-strain (Fig. 2A) and the low-virulent Dm30L-clone (Fig. 2C) maintained in triatomine condition, shows remarkable similarities above the $69 \mathrm{kDa}$ marker (1,2,4, Figs 2A, 2C), except for the absence of polypeptide 3 ( $72 \mathrm{kDa}$, pI 5.5, Fig. 2A) and small differences due to a lowering of intensity of some polypeptides below the $69 \mathrm{kDa}$ marker (7, 8 and 12 Fig. 2A). Similarly, the comparison between metacyclic forms from culture conditions shows remarkable similarities in the polypeptides below the $69 \mathrm{kDa}$ marker, despite the differences in polypeptides above the $69 \mathrm{kDa}$ marker (Figs 2B, 2D).

Western blot analysis of stage specific antigens - In order to examine the level of association between gene expression and the antigenic constitution of parasites from different host, virulence and maintenance conditions, Western blots were performed using anti-epimastigote and antimetacyclic sera prepared against virulent EP-strain parasites (Fig. 3). Epimastigote stages showed more complex and homogeneous antigenic profiles 


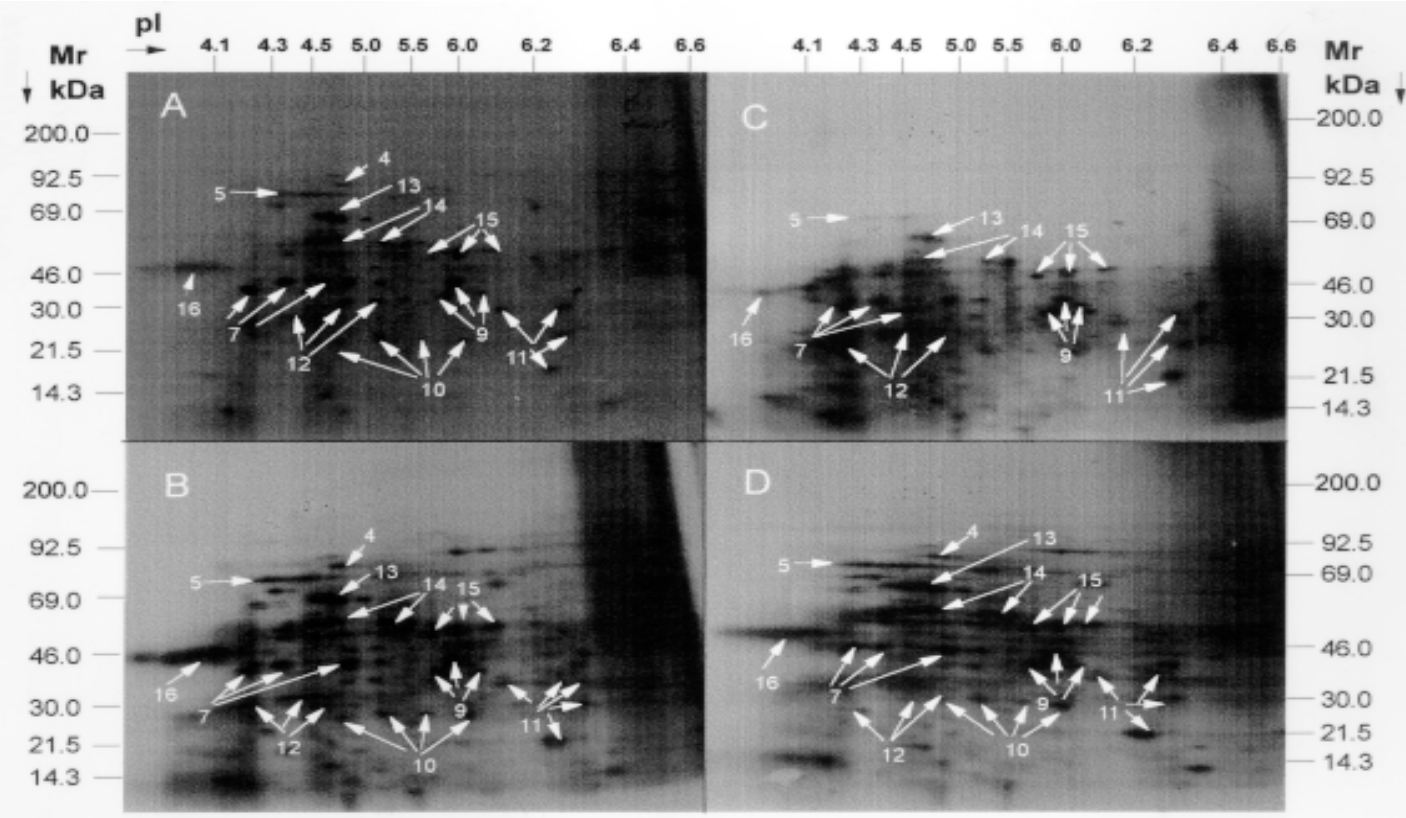

Fig. 1: analysis by two-dimensional SDS-PAGE of the polypeptides from epimastigotes of Trypanosoma cruzi (EP, Dm30L) metabolically labeled with $\left[{ }^{35} \mathrm{~S}\right]$-methionine. (A, B) EP strain and (C, D) Dm30L clone. (A, C) triatomine condition and (B, D) culture condition. Gels A, C were loaded with $600,000 \mathrm{cpm}$, and B, D with 400,000 cpm. The numbers on the top of gels (A, C) refer to the $\mathrm{pH}$ gradient of the first dimension isoelectric-focusing gel. The numbers on left and right of each figure refer to the position of the molecular weight markers.

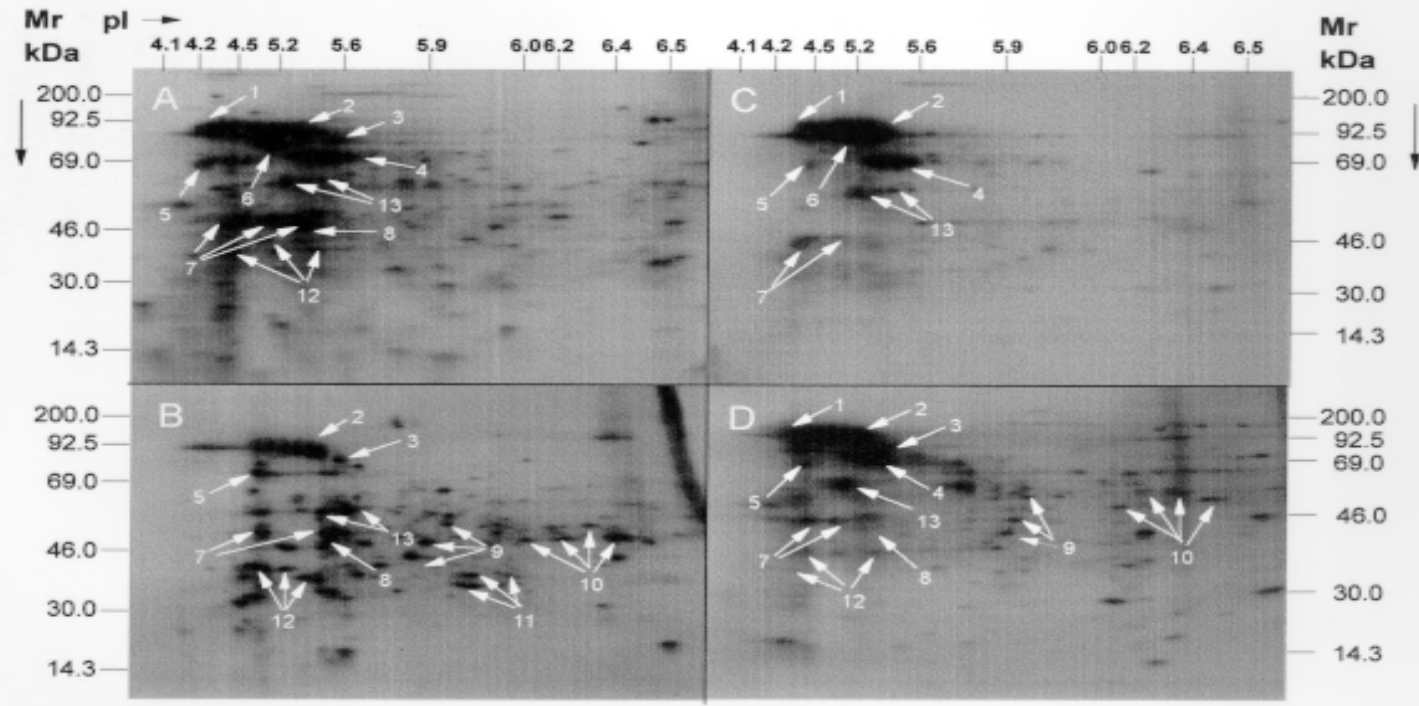

Fig. 2: analysis by two-dimensional SDS-PAGE of the polypeptides from metacyclic trypomastigotes of Trypanosoma cruzi (EP, Dm30L) metabolic labeled parasites with [35S]-methionine. (A, B) EP strain and (C, D) Dm30L clone. (A, C) triatomine condition (see Materials and Methods) and (B, D) culture condition. The number on the top of gel (A, C) refer to the pH gradient of the first dimension isoelectric-focusing gel. Each gel was loaded with $600,000 \mathrm{cpm}$. The numbers on the left and right of each figure refer to the position of the molecular weight markers. 
than metacyclic stages (lanes at left $v s$ lanes at right ). Eight antigens with $\mathrm{Mr}$ of $65,62,52,38$, $35,34,30$ and $27 \mathrm{kDa}$ were recognized by AntiEpi serum in the strain EP (lanes T, C) and in the clone Dm30L (lanes T, C). Four antigens with $\mathrm{Mr}$ of $72,55,45$ and $40 \mathrm{kDa}$ can be observed in parasites from triatomine condition (asterisks, lane T) and three antigens with $\mathrm{Mr}$ of 160,75 and $42 \mathrm{kDa}$ can be observed in parasites from culture condition (arrowhead, lane C).

The comparison of the metacyclic stages using Anti-Meta serum showed significative antigenic differences in parasites from different source maintained in triatomine condition. At least sixteen antigenic bands of different width and density with Mr of 95, 90/82 (doublet), 72, 69/67(doublet), 65, 60, 55, 51/43 (doublet), 42, 36/33 (doublet), 29 and $9 \mathrm{kDa}$ were revealed by this antiserum in the EP-strain from triatomine condition (EP lane T, Fig. 3) and only seven antigens of $\mathrm{Mr} 90 /$ 82 (doublet), 69, 65, 60,55/48, 40/38 y $9 \mathrm{kDa}$ in the Dm30L clone, maintained in the same condition (Dm30, lane T, Fig. 3). Whereas, avirulent metacyclics from culture conditions showed a overall similarity in their antigenic profiles. Seven antigens with $\mathrm{Mr}$ of $90,82 / 75,65,60,55 / 50$ (doublet), 40 and $27 \mathrm{kDa}$ were revealed by this antiserum in metacyclic forms of strain EP and in the clone Dm30L from culture conditions (EP and Dm30, lanes C, Fig. 3). However, the 72 and 47 $\mathrm{kDa}$ antigens present in metacyclic of the EP-strain (arrowhead, EP lane C) were not present in the Dm30L clone (Dm30, lane C, Fig. 3).

\section{DISCUSSION}

In this paper we have presented evidence confirming that the maintenance of $T$. cruzi in axenic culture for long time can select subpopulations less virulent than parental strains (Postan et al. 1983) and that independently of the genetic background of the parasites, the loss of virulence is associated to changes in the expression of a characteristic set of metacyclic-specific polypeptides. We also have shown that the antigens expressed by a given developmental stage, can vary according to the schedule of maintenance in the laboratory.

In $T$. cruzi, it has been reported that, despite their morphological similarities, insect-derived metacyclic forms are considerably more virulent than their culture-derived counterparts (Villalta \& Kierszenbaum 1987). In our experimental conditions, this problem was avoided by using only culture-derived metacyclic trypomastigotes. We showed that metacyclic forms of the virulent EPstrain induced in vitro from cultured-parasites recently isolated from triatomine, were as virulent for mice as insect-derived metacyclic forms (Table). However, these in vitro induced metacyclic

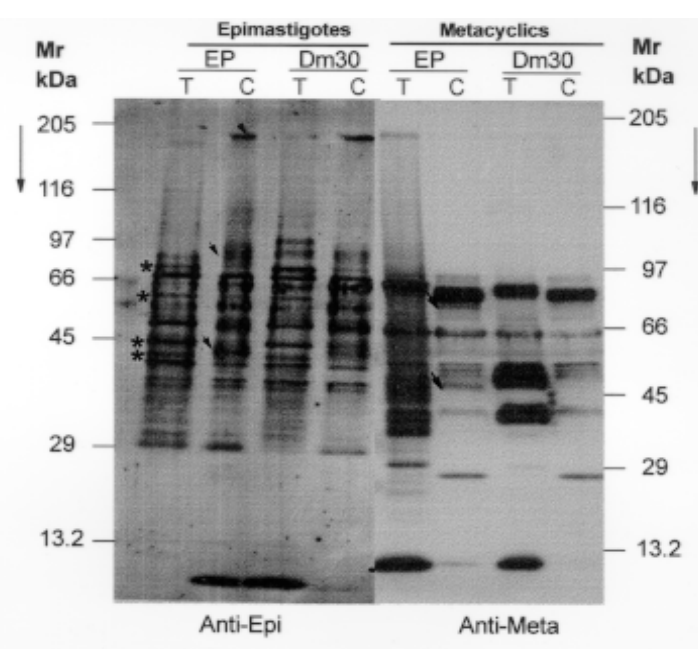

Fig. 3: Western blot analysis of total proteins from epimastigotes and metacyclic trypomastigotes of Trypanosoma cruzi (EP and Dm30L) obtained from the triatomine (T) and culture (C) conditions and incubated with anti-epimastigote (Anti-Epi) or antimetacyclics (Anti-Meta) sera (see Materials and Methods). The numbers on both sides of the figure refer to the position of the molecular weight markers.

trypomastigotes progressively loose their virulence after 78 days in culture and became almost avirulent for mice at the end of 256 days in culture. The loss of virulence of EP-strain maintained by long terms in culture could be explained by selection of a low-virulent subpopulation (Deane et al. 1984, Garcia et al. 1986). These results, taken together with previous evidences showing that metacyclic trypomastigotes of the Dm30L-clone maintained in mice condition had increased virulence (Contreras et al. 1994), and knowing that a full avirulent parasite for vertebrate host no may be maintained in nature, suggest that our maintenance conditions in laboratory is affecting intrinsically the biological properties of metacyclic trypomastigotes.

Attempting to understand at the molecular level the reasons why T. cruzi from culture condition displays full avirulence, we analyzed by electrophoresis, metabolic labeling products of epimastigotes and in vitro induced metacyclic forms. Hence, minor differences were seen in Dm30L-epimastigotes (1C, Fig. 1) may be due to its lower specific activity, a remarkable overall similarity was observed in the metabolic labeling products of epimastigotes from different hosts (EP, Dm30L) maintained in the culture conditions (1B, 1D, Fig. 1) and EP-strain epimastigotes from triatomine condition (1A, Fig. 1). This similarity 
was unexpected. One possibility to explain this result might be that we are labeling non-infective forms immediately taken from culture media. Alternatively, the labeling medium might represent a nutritional stress condition for the epimastigotes originated from a nutritional rich medium, suggesting that the epimastigotes might be mainly directed towards synthesizing the proteins necessary to survive to this condition. In contrast, metacyclic forms taken from the nutritional poor medium TAU3AAG would continue synthesizing the polypeptides characteristic of the infective stage.

If, as suggested, the metacyclic trypomastigotes protein synthesis is not altered due to labeling conditions employed, we would expected significative changes between virulent and non-virulent metacyclic forms. Our results strongly support this hypothesis. Avirulent metacyclic forms from culture conditions (Figs 2B, 2D) showed significative differences when compared to infective metacyclic trypomastigotes recently isolated from triatomine and kept in culture (Figs 2A, 2C).

The protein synthesis patterns of the virulent EP strain showed a group of acidic polypeptides with $\mathrm{Mr}$ of 69 to $92.5 \mathrm{kDa}$, and at least three of these polypeptides were absent in the protein profile of non-virulent EP strain (1, 6 and 4, Figs $2 \mathrm{~A}$ vs 2B). Similar results were observed with the lowvirulent clone Dm30L (Figs 2C vs 2D). The comparison of virulent and non-virulent protein profiles clearly indicates that the expression of metacyclic trypomastigotes (but not of epimastigotes) proteins have been altered by laboratory maintenance conditions. Iodine surface labeling showed identical surface-proteins profiles for EP-strain and Dm30L-clone from triatomine conditions whereas, several of these proteins are absent from avirulent metacyclic forms (manuscript in preparation).

The schedule of maintenance by several years the strain EP and the Dm30L clone in culture condition, obviously alters the alternability (vertebrateinvertebrate) of the parasite in nature. In terms of antigenicity, epimastigote and metacyclic stages were affected. The similarities of antigen profiles recognized for anti-epimastigotes serum in epimastigotes from culture condition (EPand Dm30, lane C, Fig. 3) seem to suggest that parasites from different host origin became antigenically homogeneous when maintained without alternate passages vertebrate/invertebrate hosts. By the other hand the maintenance of parasites in triatomine showed significative differences in the antigen profiles (EP and Dm30, lane T, Fig. 3), as expected for parasites from different sources and distinct virulences. The fact that hyperimmune serum obtained against virulent EP-metacyclic forms from triatomine condition, revealed similar antigenic profiles in the metacyclic trypomastigotes from the culture (metacyclics EP and Dm30, lane C) condition and strong differences in the antigen profiles of parasites from triatomine condition (metacyclics EP and Dm30, lane C) confirms the above suggestion. It is worth noting that the antigen profile recognized by antimetacyclic serum in parasites of the low-virulent Dm30L clone maintained in triatomine condition (Dm30 lane T, Fig. 3) resembles those from avirulent metacyclic (metacyclics lane C, Fig. 3), except to an increase in the intensity and width of some antigen bands.

The high homogeneity of the metabolic labeling products displayed by epimastigote stages, in despite of the source and maintenance condition of the parasites (Fig. 1), is in contrast with the antigenic heterogeneity showed by epimastigotes from triatomine and culture conditions (epimastigotes EP and Dm30, lanes T, C, Fig. 3). These differences can not be due to the fact that the labeling condition of epimastigotes display only high turnover proteins while Western blots reveal whole antigen of the parasites since metacyclic stage from culture conditions displayed different metabolic profiles (Figs 2B, 2D) and their antigenic profiles showed high degree of similarity (metacyclics lane T, Fig. 3). One possibility would be that stage specific antisera are able to recognize different posttranslational modifications of the antigens depending on culture conditions.

The ensemble of the data presented above suggest a close association between metacyclic specific proteins and stage-specific properties. Although, the results do not allow to establish a direct relationship between a protein and virulence of the parasites, they raise the interesting possibility that long-term maintenance of $T$. cruzi in culture might lead to the loss of a synchronous gene expression program that would occur when the parasites are maintained by successive invertebratevertebrate alternate passages.

\section{ACKNOWLEDGEMENTS}

To Dr Samuel Goldenberg for helpful discussions and for critical reading of the manuscript. To TSU Rosa Y Arteaga, Gregorio Flores, Wilmer Pineda and Rafael Garboza for technical assistance.

\section{REFERENCES}

Andrews NW, Katzin AM, Colli W 1984. Mapping of surface glycoproteins of Trypanosoma cruzi by twodimensional electrophoresis. A correlation with the cell invasion capacity. Eur J Biochem 140: 599-604.

Bonaldo MC, Souto-Padron T, Sousa W, Goldenberg, S 1988. Cell-substrat adhesion during Trypanosoma cruzi differentiation. J Cell Biol 106: 1349-1358.

Brener Z 1962. Therapeutic activity and criterion of cure 
in mice experimentally infected with Trypanosoma cruzi. Rev Inst Med Trop São Paulo 4: 389-396.

Burleigh BA, Andrews NW 1995. The mechanisms of Trypanosoma cruzi invasion of mammalian cells. Ann Rev Microbiol 49: 175-200.

Camargo ME 1966. Fluorescent antibody test for the serodiagnosis of American trypanosomiasis. Technical modification employing preserved culture forms of Trypanosoma cruzi in a slide test. Rev Inst Med Trop São Paulo 8: 227-234.

Castellani O, Ribeiro LV, Fernandes JF 1967. Differentiation of Trypanosoma cruzi in culture. J Protozool 14: 447-451.

Carneiro M, Romanha AJ, Chiari E 1991. Biological characterization of Trypanosoma cruzi strains from different zymodemes and schizodemes. Mem Inst Oswaldo Cruz 86: 387-393.

Chiari E 1974. Infectivity of Trypanosoma cruzi metacyclic trypomastigotes from culture kept in laboratory for different periods of time. Rev Inst Med Trop São Paulo 16: 61-67.

Chuenkova M, Pereira MEA 1995. Trypanosoma cruzi trans-sialidase: Enhancement of virulence in a murine model of Chagas' disease. J Exp Med 181: 1693 1703.

Contreras VT, Araque W, Delgado VS 1994. Trypanosoma cruzi metacyclogenesis in vitro. I. Changes in the properties of metacyclic trypomastigotes maintained in the laboratory by different methods. Mem Inst Oswaldo Cruz 89: 253-259.

Contreras VT, Morel CM, Goldenberg S 1985a. Stage specific gene expression precedes morphological change during Trypanosoma cruzi metacyclogenesis. Mol Biochem Parasitol 14: 83-98.

Contreras VT, Morel CM, Goldenberg S 1985c Effect of fresh guinea pig serum on $T$. cruzi protein synthesis. Anais Acad Bras Cien 51: 3-5.

Contreras VT, Salles, JM, Thomaz N, Morel CM, Goldenberg S 1985b. In vitro differentiation of Trypanosoma cruzi under chemically defined conditions. Mol Biochem Parasitol 16: 315-327.

Deane MP, Jansen AM, Mangia RHR, Gonçalves AM, Morel CM 1984. Are our laboratory "strains" representative sample of Trypanosoma cruzi population that circulate in nature? Mem Inst Oswaldo Cruz 79: 19-24.

Garcia ES, Azambuja P, Contreras VT 1984. Large scale rearing of Rhodnius prolixus and preparation of metacyclic trypomastigotes of Trypanosoma cruzi, p. 43-46. In C.M Morel, Genes and Antigens of Parasites. A Laboratory Manual, Ed. Fiocruz, Rio de Janeiro.

Garcia ES, Vieira E, Gonçalves AM, Morel CM 1986. A strain of Trypanosoma cruzi and its biochemical characterization after passage through different invertebrate host. Ann Trop Med Parasitol 80: 361-363.

Goldenberg S, Contreras VT, Salles JM, Bonaldo MC, Lima-Franco MPA, Lafaille JJ, Gonzales-Perdomo M, Linss J, Morel CM 1987. In vitro differentiating systems for the study of differential gene expression during Trypanosoma cruzi development, $\mathrm{p}$. 203-212. In N Agabian, H Goodman, N Nogueira (eds) Molecular Strategies of Parasitic Invasion, UCLA Symp. Molec. Cell Biology, New Series,
Vol. 42, Alan R Liss Inc., New York.

Gomes ML, Romanha AJ, Gonçalves AM, Chiari E 1991. Stability of isoenzyme and kinetoplast DNA (k-DNA) patterns in successively cloned Trypanosoma cruzi populations. Mem Inst Oswaldo Cruz 86: 379-385.

Laemmli UK 1970. Cleavage of structural proteins during the assembly of the head of bacteriophage T4. Nature 227: 680-685.

Laskey R, Mills AD 1977. Enhanced autoradiographic detection of ${ }^{32} \mathrm{P}$ and ${ }^{125} \mathrm{I}$ using intensifying screens and hypersensitized film. FEBS Lett 82: 314-316.

Leskawa KC, Hicerson S, Cambron LD 1993. A quantitative method for assessing protein abundance using enhanced chemiluminiscence. BioTechniques 15: 790-794.

O'Farrell PH 1975. High-resolution two-dimensional electrophoresis of proteins. J Biol Chem 250: 4007 4021.

Postan M, Dvorak JA, McDaniel JP 1983. Studies of Trypanosoma cruzi in inbred mice. I - A comparison of the course of infection of $\mathrm{C} 3 \mathrm{H} / \mathrm{HEN}^{-}$mice with two clones isolated from a common source. Am J Trop Med Hyg 32: 497-506.

Ramirez MI, Ruiz RC, Araya JE, Franco da Silveira F, Yoshida N 1993. Involvement of the stage-specific 82-kilodalton adhesion molecule of Trypanosoma cruzi metacyclic trypomastigotes in host cell invasion. Infect Immunol 61: 3636-3641.

Ruiz RC, Rigoni VL, Gonzalez J, Yoshida N 1993. The 35/50 kDa surface antigen of Trypanosoma cruzi metacyclic trypomastigote, an adhesion molecule involved in host cell invasion. Parasit Immunol 15: 121-125.

Sousa MA 1983. Surface electrical charge of bloodstream trypomastigotes of Trypanosoma cruzi strains. Mem Inst Oswaldo Cruz 78: 497-500.

Spector T 1981. Refinement of the Comassie blue method of protein quantitation. Anal Biochem 86: 142-146.

Schenkman RPF, Vandekerckhove F, Schenkman S 1993. Mammalian cell sialic acid enhances Trypanosoma cruzi invasion. Infect Immun 61: 898-902.

Towbin H, Stachelin T, Gordon J 1979. Electrophoretic transfer of proteins from polyacrylamide gels to nitro-cellulose sheets: Procedure and some applications. PNAS 79: 4350-4354.

Vermelho AB, Meirelles MNL 1994. Sialoglycoconjugates in Trypanosoma cruzi-host cell interaction: Possible biological model - A review. Mem Inst Oswaldo Cruz 89: 69-79.

Villalta F, Kierszenbaum F 1987. Insect-borne and culture-derived metacyclic Trypanosoma cruzi: Differences in infectivity and virulence. Am J Trop Med Hyg 36: 529- 532.

Yoshida N, Blanco SA, Araguth MF, Russo M, Gonzalez G 1990. The stage specific 90-kiloDalton surface antigen of metacyclic trypomastigotes of Trypanosoma cruzi. Mol Biochem Parasitol 39: 39-46.

Yoshida N, Dorta ML, Ferreira AT, Oshiro MEM, Mortara RA, Acosta-Serrano A, Favoret Jr S 1997. Removal of sialic acid from mucin-like surface molecules of Trypanosoma cruzi metacyclic trypomastigotes enhances parasite-host cell interaction. Mol Biochem Parasitol 84: 57-67. 